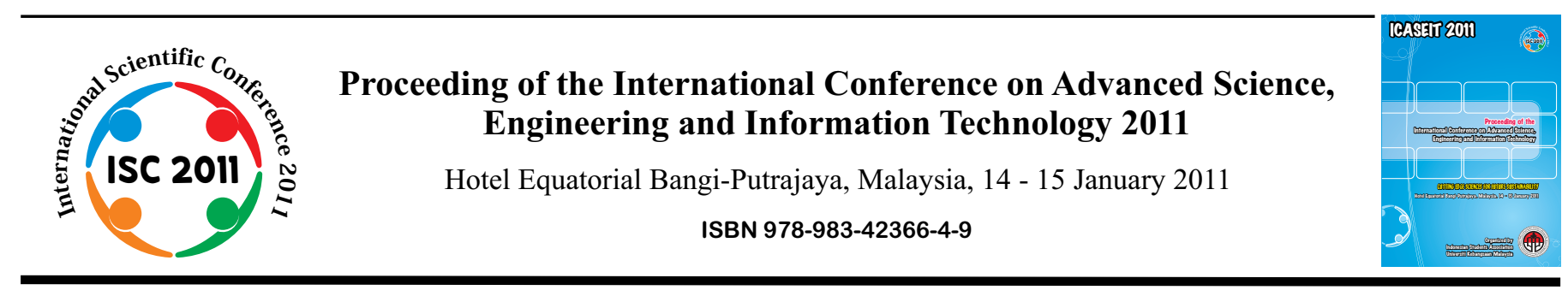

\title{
Myocardial Motion Estimation: An Evaluation of Optical Flow Computation Techniques on Echocardiographic Images
}

\author{
Slamet Riyadi ${ }^{\#}$, Mohd Marzuki Mustafa ${ }^{\#}$, Aini Hussain" ${ }^{\#}$ Oteh Maskon ${ }^{*}$ and Ika Faizura Mohd Nor ${ }^{*}$ \\ \# Department of Electrical, Electronic and System Engineering \\ Faculty of Engineering and Built Environment, Universiti Kebangsaan Malaysia, Bangi 43600 Malaysia \\ E-mail: \{riyadi, marzuki, aini\}@eng.ukm.my \\ *Cardiac Care Unit \\ Medical Center, Universiti Kebangsaan Malaysia, Kuala Lumpur 56000 Malaysia \\ E-mail:auajwad@yahoo.com,azuzayz@yahoo.ie
}

\begin{abstract}
The use of image processing technique for cardiac motion analysis has been an active research in the past decade. The estimation of myocardial motion eases the cardiologist in diagnosing cardiac abnormalities. In term of movement analysis, optical flow is the most popular technique that has been used by researchers. This paper describes the implementation and evaluation of three optical flow computation techniques to estimate the myocardial motion using echocardiographic images. The three techniques are the global smoothness method (GSM), the local smoothness method (LSM) and warping technique (WT). Optical flow field is computed based on healthy cardiac video on parasternal short axes view. These techniques look promising since the optical flow fields can be utilized to estimate the myocardial movement and comply with its true movement. The performances of each technique in terms of the direction, homogeneity and computing time, are also discussed.
\end{abstract}

Keywords - Myocardial motion, optical flow, echocardiography images, global smoothness, local smoothness, warping.

\section{INTRODUCTION}

Myocardial infarction, or known as heart attack, is the leading cause of death all over the world. Early detection of coronary heart disease (CHD) can be performed via anomaly motion detection of the left ventricular (LV) of the heart. LV motion analysis from clinical image sequences has been an active research area over the past decade. Various techniques have been proposed by researcher such as grids of intersection technique based on elastic non-rigid structure [1][7] and Bayesian estimation framework [8][6]. Ledesma et al developed a new spatio-temporal nonrigid registration to estimate the displacement fields from two-dimensional ultrasound sequence of the hearts [5].

In term of movement analysis in computer vision, optical flow is a popular technique to determine the displacement field of two consecutive images. Various improvements and modifications have been done by researcher whether in theory or application approaches of the optical flow.
Different methods of optical flow computation have been developed on various human related real problems. In this paper, we implement and evaluate three different optical flow computation techniques to estimate the LV motion. The techniques used are the global smoothness method (GSM), the local smoothness method (LSM) and warping technique (WT). The evaluation is performed on 2D echocardiographic images since the 2D imaging technique is commonly used in clinical practice in Malaysia.

\section{MYOCARDIAL VIEW AND ITS MOVEMENT}

The Cardiac Imaging Committee of the Council on Clinical Cardiology of the America Heart Association has released a standardized myocardial segmentation and nomenclature for tomography imaging of the heart [5]. In this paper, the evaluation of myocardial movement is focused only on a parasternal short axes (PSAX) view as shown in Fig. 1. The LV appears as a cavity in a centre surrounded by six myocardial segments where as normal 
cardiac appearance is indicated by a uniform displacement of myocardial segments. They move in radial forward and backward directions with respect to the centre of cavity, which describe the cardiac profile of systole and diastole. During one time period, the cavity size will shrink progressively during systole and it is followed by a brief instance of diastole that recovers the cavity [1]. Thus the velocity of each segment is computed accurately during one complete cycle of myocardial movement.

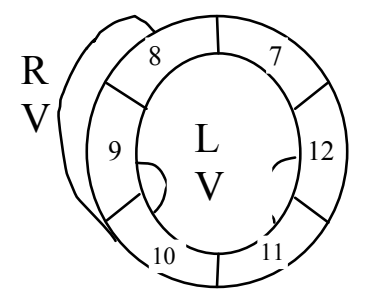

$$
\begin{aligned}
& \text { LV }=\text { left ventricular } \\
& \text { RV }=\text { right ventricular } \\
& 7=\text { mid anterior } \\
& 8=\text { mid anteroseptal }
\end{aligned}
$$

$$
\begin{aligned}
& 9=\text { mid inferoseptal } \\
& 10=\text { mid inferior } \\
& 11=\text { mid inferolateral } \\
& 12=\text { mid anterolateral }
\end{aligned}
$$

Fig. 1 PSAX view of echocardiography image and its segments

\section{Methodology}

The methodology involves several tasks such as the collection of echo-cardiographic images and the computation of optical flow field using three selected techniques. In this work, PSAX view of echocardiographic video is obtained from the patient with healthy cardiac in the rest condition (baseline). Then, the motion vector is represented by the optical flow between two consecutive frames of echocardiographic images. Finally, the performances of each technique in terms of the direction, homogeneity and computing time, are also discussed.

\section{OPTICAL FLOW COMPUTATION TECHNIQUES}

Optical flow field is computed by assuming that the intensity of the object remains constant from the initial point of $\mathrm{I}(\mathrm{x}, \mathrm{y}, \mathrm{t})$ towards the latest position of $\mathrm{I}(\mathrm{x}+\delta \mathrm{x}, \mathrm{y}+\delta \mathrm{y}, \mathrm{t}+\delta \mathrm{t})$. Thus, the intensity constraint of a point is represented by

$I(x, y, t)=I(x+\delta x, y+\delta y, t+\delta t)$

Within small values of $\delta x, \delta y$ and $\delta$ t, the intensity constraint can be represented using the 1st order of the Taylor series expansion as,

$$
I(x+\delta x, y+\delta y, t+\delta)=I(x, y, t)+\frac{\partial I}{\partial x} \delta x+\frac{\partial I}{\partial y} \delta y+\frac{\partial I}{\partial t} \delta
$$

Simplifying equation (1) and (2), the basic constraint of the optical flow equation can be expressed as

$$
I_{x} v_{x}+I_{y} v_{y}+I_{t}=0, \quad \text { or } I_{\mathrm{x}} u+I_{\mathrm{y}} v+I_{\mathrm{t}}=0
$$

The intensity constancy assumption is very sensitive to brightness changes that contrarily often appear in natural cases. Therefore, it is important to introduce other assumptions to solve the estimation problem.

\section{A. Global smoothness method}

Horn-Schunck in [3] proposed global smoothness error (ES) and data error (ED) defined as

$$
\begin{aligned}
& E_{s}=\iint_{D}\left(u_{x}^{2}+u_{y}^{2}\right)+\left(v_{x}^{2}+v_{y}^{2}\right) d x d y \\
& E_{D}=\iint_{D}\left(I_{x} u+I_{y} v+I_{t}\right)^{2} d x d y
\end{aligned}
$$

To determine the optical flow vector (u, v), they computed an iterative solution to minimize both errors following the equation ED $+\lambda$ ES where $\lambda$ represents the determined smoothing factor.

\section{B. Local smoothness method}

In [2], Lukas-Kanade developed another approach to solve the constraint equation. By assuming that velocity $(\mathrm{u}, \mathrm{v})$ in a small neighborhood is constant, they proposed a weighted least-square in the optical flow computation for each small spatial neighborhood by minimizing equation

$$
\sum_{x, y \in A} W^{2}(x, y)\left[\nabla I(x, y, t) \cdot \vec{v}+I_{t}(x, y, t)\right]^{2}
$$

The solution is given by

$$
\begin{gathered}
\vec{v}=\left[A^{T} W^{2} A\right]^{-1} A^{T} W^{2} \vec{b} \\
\text { where } \quad A=\left[\nabla I\left(x_{1}, y_{1}\right), \ldots, \nabla I\left(x_{N}, y_{N}\right)\right] \\
W=\operatorname{diag}\left[W\left(x_{1}, y_{1}\right), \ldots, W\left(x_{N}, y_{N}\right)\right] \\
\vec{b}=-\left(I_{t}\left(x_{1}, y_{1}\right), \ldots, I_{t}\left(x_{N}, y_{N}\right)\right)
\end{gathered}
$$

\section{The warping technique}

Brox et al [7] summarized that using the three assumptions, namely intensity constancy, gradient constancy and smoothness assumption, velocity vectors $\mathrm{u}$ and $\mathrm{v}$ can be determined by minimizing the total energy $\mathrm{E}(\mathrm{u}, \mathrm{v})$, such that

$$
E(u, v)=E_{\text {Data }}+\alpha E_{\text {Smooth }}
$$

where $>0$ is the regularization parameter. The derivation of intensity and gradient constancy assumption can be expressed as:

$$
E_{\text {Data }}(u, v)=\int_{\Omega} \Psi\left(|I(x+w)-I(x)|^{2}+\gamma|\nabla I(x+w)-\nabla I(x)|^{2}\right) d x
$$

To overcome the aperture problem, researchers introduce the smoothness assumption. Optical flow computation is not only based on a single pixel displacement but it also considers interactions between neighbouring pixels. This smoothness criterion is computed using total variation of piecewise smooth flow field which can be expressed as

$$
E_{\text {Smooth }}(u, v)=\int_{\Omega} \Psi\left(\left|\nabla_{3} u\right|^{2}+\left|\nabla_{3} v\right|^{2}\right) d x
$$

The minimization computation is performed by numerical approximation combined with the down sampling strategy.

\section{Optical Flow COMPUTATION TECHNIQUES}

The above summarized optical flow computation procedures are used for the myocardial motion estimation based on echocardiographic images. Fig. 2 shows the myocardial movement sequences where Fig. 2 (a) to (d) indicate the diminishing motion of LV. These images were taken from a normal cardiac motion with PSAX view of the LV. The LV appeared in the centre of the image as a small, rounded structure with an echo-free cavity. This cavity size should diminish in a concentric manner during systole, but not to be obliterated [1].

Fig. 4 (a), (c) and (e) show the optical flow field results for the myocardial motion estimation of two consecutive 
images of Fig. 2(c) and (d) using three different optical flow computation methods. The arrows represent the displacement of the pixels and their directions. The different colors of arrows represent the speed of displacements that change gradually from red to blue indicating a large to small displacement representation. Based on the general visual appearance of the computed optical flow field, all methods performed an accurate estimation of myocardial motion. The movement of myocardial is represented by the red arrows and these arrows diminish as the move towards the centre of the cavity.

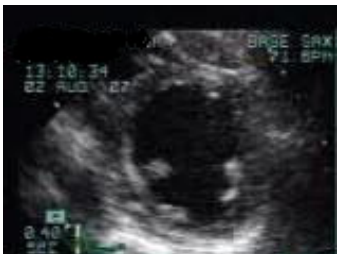

(a)

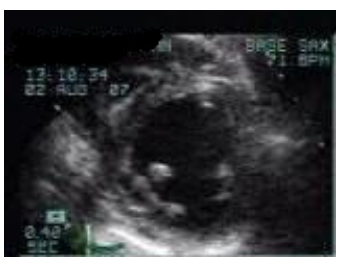

(c)

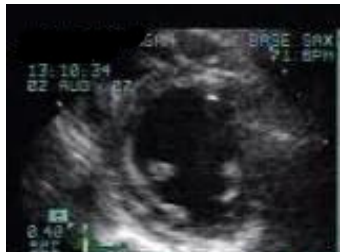

(b)

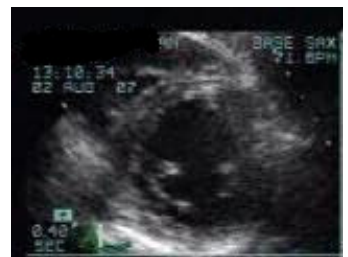

(d)
Fig. 2 (a) to (d) Clinical echocardiography images showing the myocardial movement sequences of the LV as the cavity diminishes during systole.

In this study, we also computed the direction angle of optical flow respect to the center of LV as illustrated in Fig. 3. The direction angle $\alpha=0$ means that the optical flow move towards to the center of cavity. The distribution of direction angle of each computed optical flow in Fig. 4 (a), (c) and (e) is depicted in Fig. 4 (b), (d) and (f), respectively. The distribution shows that the direction angle of computed optical flow has a similar pattern, i.e. move towards to the centre. This is in total agreement with the understanding of cardiac sequence image that during systole, the cavity size should diminish in a concentric manner.

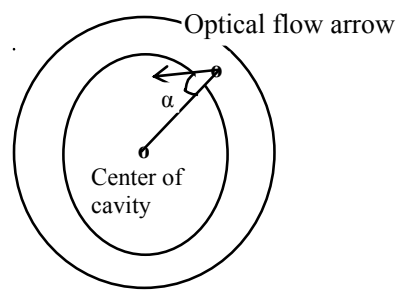

$\alpha=$ Direction angle respect to the center of cavity

Fig. 3 The illustration of direction angle of optical flow respect to the center of cavity

The performance of each technique is observed using the standard deviation of direction

$S D_{\theta}=\frac{\sum \sigma_{\theta}|(u, v)|}{\sum|(u, v)|}$ where $\sigma_{\theta}$ is the standard deviation of optical flow angle and $|(u, v)|$ is the magnitude of optical flow. The computed $S D_{\theta}$ are tabulated in Table 1, which represent the homogeneity of the arrow direction. According to $S D_{\theta}$, it can be seen that the computed optical flow of the WT was the smoothest in term of the directional property in which the directions of neighbouring pixels are homogeneous and truly comply with the true movement of myocardial segments. On the other hand, the WT was not able to produce a sharp boundary of the myocardial segment.

When applied onto an image of size $384 \times 287$, the WT took the longest computing time of more than 7 seconds as compared to both LSM (3.01 seconds) and GSM (0.48 seconds). This is due to the smoothing iteration process involved in the WT. Compromising between both performances, the finest optical flow was obtained via the LSM or local smoothness method as shown in Figure 3(b).

TABLE I

PERFORMANCE OF OPTICAL FLOW TECHNIQUES

\begin{tabular}{lccc}
\hline \hline & GSM & LSM & WT \\
\hline Standard deviation of direction & 82.36 & 38.35 & 6.22 \\
Computation time (seconds) & 0.48 & 3.01 & 7.30 \\
\hline \hline
\end{tabular}

\section{CONCLUSION}

The implementation and evaluation of three different optical flow computation techniques to estimate myocardial motion using echocardiographic images are described. The findings confirmed that the optical flow field has similar characteristics of the true myocardial movements in which the cavity diminishes during systole in a concentric manner. As such, it can be concluded that optical flow field can be used as a true reflection in estimating and visualizing the myocardial motion since the optical flow fields are in total agreement of the true myocardial motion.

\section{REFERENCES}

[1] B. Anderson, "Echocardiography: The Normal Examination of Echocardiographic Measurements", Blackwell Publishing, 2002, "Chapter 2: The 2-dimensional echocardiographic examination".

[2] B. D. Lucas and T. Kanade, "An interative image registration technique with application in stereo vision", Proceeding of IJCAI, 1981.

[3] B. K. P. Horn,. and B.G. Schuck., "Determining Optical Flow," Artificial Intelligent, vol 9, pp 185-203, 1981

[4] L. Axel, R. Goncalves, and D. Bloomgarden, "Regional heart wall motion: Two-dimensional analysis and functional imaging with MR images", Radiology 183, pp. 745-750, 1992.

[5] M. J. Ledesma-Carbayo, J. Kybic, M. Desco, A. Santos, M. Suhling, P. Hunziker, and M. Unser, "Spatio-temporal nonrigid registration for ultrasound cardiac motion estimation", IEEE Transcation on Medical Imaging 24:9, pp. 1113-1126, 2005.

[6] M. Qazi, G. Fung, S. Krishnan, R. Rosales, H. Steck, R. B. Rao, D. Poldermans, D. Chandrasekaran, "Automated heart wall motion abnormality detection from ultrasound images using Bayesian networks", Proceeding of IJCAI 07, pp. 519-525, 2007.

[7] Petland and B. Horowitz, "Recovery of non-rigid motion and structure", IEEE Transaction on Pattern Analysis and Machine Intelligent 13, pp. 730-742, 1991.

[8] T. Brox, A. Bruhn, N. Papenberg and J. Weickert. "High Accuracy Optical Flow Estimation Based on a Theory for Wrapping," Proc. 8th European Conference on Computer Vision, Springer LNCS 3024, vol 4, pp 25-36, 2004

[9] X. Papademetris, A. J. Sinusas, D. P. Dione, and J. S. Duncan, “3D cardiac deformation from ultrasound images", Proceeding of MICCAI 99, pp 420-429, 1999. 


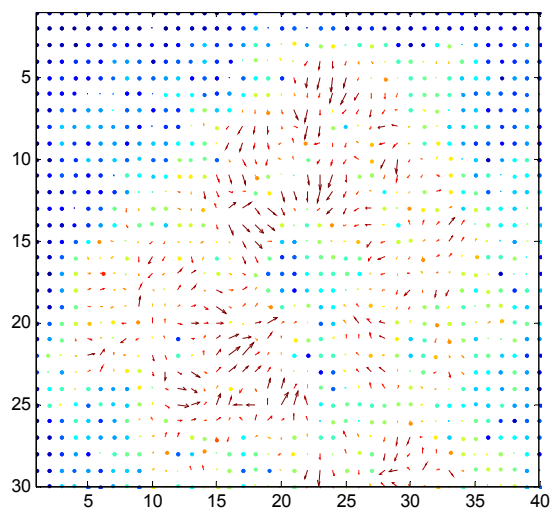

(a)



(c)

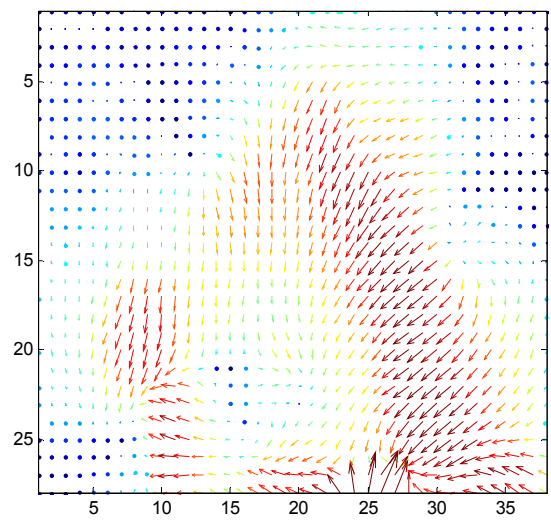

(e)

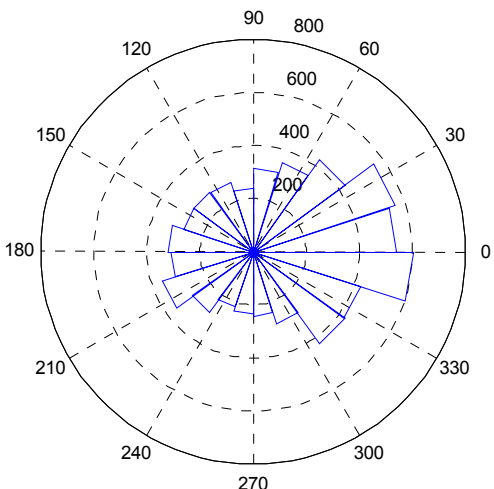

(b)



(d)



(f)

Fig. 4 The computed optical flow field of myocardial motion of two consecutive images of Fig. 2(c) and (d) using (a) GSM, (c) LSM and (e) WT, and their distribution of direction angle respect to the center of cavity (b), (d) and (f), respectively. 